\title{
Design of Sharp FIR Filters with Prescribed Group Delay
}

\author{
Ju-Hong Lee and Charng-Kann Chen \\ Department of Electrical Engineering
National Taiwan University \\ National Taiwan University \\ Taipei, Taiwan, R. O. C.
}

\begin{abstract}
In this paper, we propose a method for synthesizing a sharp FIR digital filter with prescribed group delay. In general, the filter length of an FIR filter is inversely proportional to the filter's transition width. Hence, for a sharp linear phase FIR filter the complexity would be very high and the group delay would be very large. Based on a weighted least squares (WLS) algorithm, we extend the frequency response masking approach introduced in [3] to the complex domain to significantly reduce the required complexity and group delay for synthesizing a sharp FIR filter. The effectiveness of the proposed method is demonstrated by design examples.
\end{abstract}

\section{Introduction}

Linear phase FIR filters are known to have some very desirable features like guaranteed stability, free from limit cycles and phase distortion, and low coefficient sensitivity. The drawback of them is the large number of arithmetic operations required in the implementation. This problem becomes more serious when the filter has a very tigh specification with a very narrow transition-width. Another disadvantage of linear phase FIR filter is its unacceptable long signal delay when used to synthesize a narrow transitionband filter. These two disadvantages are owing to the facts that the order of an FIR digital filter is inversely proportional to the transition-width and the group delay of a linear phase FIR filter is equal to one half of the filter's order.

Considering the design techniques for reducing the complexity of sharp FIR filters, many linear phase computationally efficient realizations have been reported in the literature [1]--[5]. Interpolated FIR (IFIR) filter [1] is a computationally efficient filter structure which composes of a cascade of two FIR filters $F\left(z^{M}\right)$ and $G(z)$. The resulting system has bandwidth and transition-width both are $1 / M$ of those of $F(\omega)$. This scheme is very effective in synthesizing narrow-band and wide-band filters. Recently, some general structures for the design of IFIR filters have been further investigated in [2]. However, these approaches are not suitable for designing sharp filters with arbitrary bandwidth. This shortcoming has been alleviated by using the concept of complementary filters in [3], where a generalized IFIR structure and a frequency response masking approach are proposed to design sharp FIR filters with arbitrary bandwidth. The success of the above methods are owing to the effective exploiting the existing strong correlation between the neighboring impulse response value of filters. Another interesting scheme to reduce the cost of implementation of an FIR filter is to design the filter by interconnecting a number of identical subfilters with the aid of a few additional adders and multipliers. Such approach was first proposed in [4]. Recently, [5] gave a thorough treatment to this filter structure and several approximation problems were considered. The advantage of this structure is that we only need to design a small order subfilter and several tap coefficients to synthesize a sharp FIR filter.

Although the above methods can significantly reduce the complexity of a sharp FIR filter, the group delay of the filter is still very large. It is because that all of the above methods concern the linear phase design only and we cannot further to reduce the minimum order required to satisfy a given specification. In order to design filters which have less delay than linear phase filters and have approximately constant delay in the filter passband, the problem of complex frequency approximation problem must be considered [6].
In this paper, based on a recently developed weighted least square (WLS) algorithm [7] and a modified discrete optimization procedure, we extend the frequency response masking approach 3 ] to the complex domain to solve the above two design problem$\mathrm{s}$. The filter structure we used is shown in Fig. 1. During the design process, we first search the optimal interpolating factor $M$ to minimize a complexity measure of the overall system. Then, the design specifications of the two masking filters $F_{M A}(z)$ and $F_{M C}(z)$ are determined such that we can use the WLS algorithm for the design. Finally, we again use the WLS algorithm to design the shaping filter $H_{p}\left(z^{M}\right)$ to equalize the overall response. The reason why we use the WLS algorithm to design these three subfilters is that the WLS approach is efficient in solving the complex frequency approximation problem. It is shown that by using the proposed method, sharp FIR filters with prescribed group delay can be easily designed.

This paper is organized as follows. Section II describes the filter structure and states the associated design problems. In Section III, we present a method for searching the interpolating factor $M$ to minimize a complexity measure of the overall system. Next, we determine the specifications of $F_{M A}(z)$ and $F_{M C}(z)$, and describe a design method for these two filters in Section IV. With the two designed masking filters, we state a method to design the shaping filter $H_{p}\left(z^{M}\right)$ in Section V. Several design examples for demonstrating the effectiveness of the proposed method are included in Section VI. Finally, we conclude this paper in Section VII.

\section{Problem Formulation}

Let $H_{p}(z)$ be a lowpass FIR filter with passband group delay $G_{p}$, peak passband ripple $\delta_{1}$, and peak stopband ripple $\delta_{2}$. Let another filter $H_{c}(z)$ be defined as

$$
H_{c}(z)=z^{-G_{p}}-H_{p}(z),
$$

then it is a highpass FIR filter with passband group delay $G_{p}$, peak passband ripple $\delta_{2}$, and peak stopband ripple $\delta_{1}$. It is obvious that $\left|H_{p}\left(e^{j \omega}\right)+H_{c}\left(e^{j \omega}\right)\right|=1$, therefore $H_{p}(z)$ and $H_{c}(z)$ are complementary pair [3]. If we further constrain the group delay value $G_{p}$ to be an integer, then $H_{p}(z)$ and $H_{c}(z)$ can be implemented simultaneously by using the identical tapped delay line with an extra subtractor.

The proposed structure is shown in Fig. 1. This structure has the transfer function

$$
H(z)=H_{p}\left(z^{M}\right) F_{M A}(z)+H_{c}\left(z^{M}\right) F_{M C}(z) .
$$

One possible magnitude response operation of Fig. 1 for the case $M=4$ is shown in Fig. 2. From Fig. 2, we observe that if we properly select the two masking filters $F_{M A}(\omega)$ and $F_{M C}(\omega)$ then the transition-width of $H(\omega)$ would be $1 / M$ of that of $H_{p}(\omega)$. Besides, if we further design $F_{M A}(\omega)$ and $F_{M C}(\omega)$ to have the same passband group delay $G_{m}$, then the overall system is an FIR system with constant passband group delay $M G_{p}+G_{m}$. From these two observations, we ensure that the proposed structure can synthesize a sharp FIR filter with prescribed passband group delay.

Hence, the design problem can be stated as follows: Given the desired specifications, like the passband edge $\omega_{p}$, stopband edge $\omega_{s}$, passband ripple $\delta_{p}$, stopband ripple $\delta_{s}$, and passband group delay 
$G$, the design task is to find the three subfilters $H_{p}(z), F_{M A}(z)$, and $F_{M C}(z)$ to make $H(z)$ of (2) meet the desired specifications.

To design such system, we must first determine the interpolating factor $M$ and all the design specifications of $H_{p}(\omega), F_{M A}(\omega)$, and $F_{M C}(\omega)$. These specifications include the desired responses, bandedges, and allowable band-ripples of the three subfilters. In the following sections, we describe how to determine these design specifications and design these subfilters.

\section{Determination of $\mathbf{M}$}

The value of $M$ directly relates to the complexity of the overall system. Hence, $M$ is determined to make the complexity of $H(z)$ achieve to a minimum. Basically, the complexity of $H(z)$ can be defined as the sum of the filter lengths of $H_{p}(z), F_{M A}(z)$, and $F_{M C}(z)$. One way to measure the length of each subfilter is to calculate the corresponding transition-width. Once the transitionwidths of these subfilters are obtained, we can easily define a complexity measure of the overall system.

For some fixed $M$, there are two possible situations. The transition band of $H(\omega)$ can be mainly determined by either $H_{p}(M \omega)$ or $H_{c}(M \omega)$. We call these two situations as Case I and Case II, respectively. Let $\theta$ and $\phi, \omega_{A p}$ and $\omega_{A s}$, and $\omega_{C p}$ and $\omega_{C s}$ be the passband edge and the stopband edge of $H_{p}(\omega), F_{M A}(\omega)$, and $F_{M C}(\omega)$, respectively. The relationships of these quantities are listed in Table I.

From Table I, we can see that for each case, the transitionwidths of $H_{p}(\omega), F_{M A}(\omega)$, and $F_{M C}(\omega)$ are $\phi-\theta, \frac{2 \pi-(\phi+\theta)}{M}$, and $\frac{\phi+\theta}{M}$, respectively. Thus, we can define a complexity measure as

$$
C(M)=\frac{1}{\phi-\theta}+\frac{M}{2 \pi-(\phi+\theta)}+\frac{M}{\phi+\theta} .
$$

Obviously, $C(M)$ is the sum of the reciprocals of the three transition widths. Although the truly estimate of the complexity of $H(z)$ is a function which is more complicated than the proposed one, from our experience, (3) performs well enough for most of the cases. For some $M$, we first determine whether it belongs to Case I or Case II, and then we calculate the $\phi$ and $\theta$ by using the formulas in Table I. Substituting $\phi, \theta$, and $M$ into (3), we can obtain the corresponding $C(M)$. A good choice of $M$ can be obtained by calculating $C(M)$ for each $M$ and then selecting the $M$ which corresponds to the smallest $C(M)$. Using the selected $M$, we can uniquely determine the bandedges of the three subfilters.

Next, we consider a method for choosing the suitable group delay value $G_{p}$ and $G_{m}$. Let $b_{p}$ be $\phi-\theta$ and $b_{m}$ be $\min \left(\frac{\phi+\theta}{M}, \frac{2 \pi-(\phi+\theta)}{M}\right)$, where $M$ is the value which makes (3) achieve a minimum. Basically, a filter with smaller transition-width has a larger group delay. We further determine the group delay value $G_{p}$ and $G_{m}$ by solving the following linear equations:

$$
\left\{\begin{array}{l}
G_{p} b_{p}=G_{m} b_{m} \\
M G_{p}+G_{m}=G
\end{array}\right.
$$

The obtained $G_{p}$ is not a integer in general. However, for the sake of easy implementation, we must fix the $G_{p}$ at an integer value. Hence, we can determine the final value of $G_{p}$ and $G_{m}$ as

$$
G_{p}^{\prime}=\left\lceil G_{p}\right\rceil \text {, and } G_{m}=G-M G_{p}^{\prime},
$$

respectively. The remaining undetermined specifications for these subfilters are the allowable band-ripples of each subfilter. However; there is a very subtle relationship between the given $\delta_{p}, \delta_{\text {, }}$ and the allowable band-ripples of each subfilter. This subtle relationship can not be explicitly demonstrated without an analysis of the approximation error $\delta(\omega)$ of $H(\omega)$. In the following section; we give a simple analysis for $\delta(\omega)$, and show how to use the analysis result to design the two masking filters.

\section{Design of $F_{M A}(z)$ and $F_{M C}(z)$}

Let the desired response of $H_{p}(M \omega)$ be $D_{p}(\omega)=e^{-j \omega M G_{P}}$ in the passband of $H_{p}(M \omega)$ and $D_{p}(\omega)=0$ in the stopband of $H_{p}(M \omega)$, and the approximation error of $H_{p}(M \omega)$ be $\delta_{H_{p}}(\omega)$. Let $D_{M A}(\omega)$ and $\delta_{M A}(\omega)$, and $D_{M C}(\omega)$ and $\delta_{M C}(\omega)$ be the de- sired response and approximation error of $F_{M A}(\omega)$ and $F_{M C}(\omega)$, respectively. In the passbands of $F_{M A}(\omega)$ and $F_{M C}(\omega)$, both $D_{M A}(\omega)$ and $D_{M C}(\omega)$ are $e^{-j \omega G_{m}}$. In the stopbands of $F_{M A}(\omega)$ and $F_{M C}(\omega)$ both $D_{M A}(\omega)$ and $D_{M C}(\omega)$ are 0 . Moreover, let $D(\omega)$ and $\delta(\omega)$ be the desired response and approximation error of $H(\omega)$. In the passband and stopband of $H(\omega), D(\omega)$ is $e^{-j \omega\left(M G_{p}+G_{m}\right)}$ and 0 , respectively. Using (2), we have

$$
\begin{aligned}
& D(\omega)+\delta(\omega)=\left[D_{M A}(\omega)+\delta_{M A}(\omega)\right]\left[D_{p}(\omega)+\delta_{H p}(\omega)\right]+ \\
& {\left[D_{M C}(\omega)+\delta_{M C}(\omega)\right]\left[e^{-j \omega M G_{p}}-D_{p}(\omega)-\delta_{H p}(\omega)\right] .}
\end{aligned}
$$

For the situation of Case I as shown in Fig. 2. The situation of Case II can be analyzed in the similar way. Considering the approximation error $\delta(\omega)$ of $H(z)$ in the frequency range $\left[0, \frac{2 m m x-\beta}{M}\right]$. In this region, both $D_{M A}(\omega)$ and $D_{M C}(\omega)$ are $e^{-j \omega G_{m}}$, and $D(\omega)$ is $e^{-j \omega\left(M G_{p}+G_{m}\right)}$. Substituting these quantities into $(6)$, we have

$$
\begin{aligned}
\delta(\omega)= & \delta_{M C}(\omega) e^{-j \omega M G_{\mathrm{p}}}+D_{p}(\omega)\left[\delta_{M A}(\omega)-\delta_{M C}(\omega)\right]+ \\
& \delta_{H_{p}}(\omega)\left[\delta_{M A}(\omega)-\delta_{M C}(\omega)\right]
\end{aligned}
$$

Ignoring the second order term, we have

$$
\delta(\omega) \simeq \delta_{M C}(\omega) e^{-j \omega M G_{p}}+D_{p}(\omega)\left[\delta_{M A}(\omega)-\delta_{M C}(\omega)\right] .
$$

When $D_{p}(\omega)=e^{-j \omega M G_{p}}$, we have

$$
\delta(\omega) \simeq \delta_{M A}(\omega) e^{-j \omega M G_{p}} .
$$

When $D_{p}(\omega)=0$, we have

$$
\delta(\omega) \simeq \delta_{M C}(\omega) e^{-j \omega M G_{p}} .
$$

For $D_{p}(\omega)$ in the transition band, we can express $D_{p}(\omega)$ as the value $a e^{-j \omega M G_{p}}$, where $a$ is a real positive number and $0<a<1$. Replacing $D_{p}(\omega)$ by $a e^{-j \omega M G_{p}}$ in (8), we have

$$
\delta(\omega) \simeq(1-a) \delta_{M C}(\omega) e^{-j \omega M G_{p}}+a e^{-j \omega M G_{p}} \delta_{M A}(\omega),
$$

and hence

$$
|\delta(\omega)| \leq(1-a)\left|\delta_{M C}(\omega)\right|+a\left|\delta_{M A}(\omega)\right| .
$$

(12) shows that $|\delta(\omega)|$ is a convex combination of $\left|\delta_{M C}(\omega)\right|$ and $\left|\delta_{M A}(\omega)\right|$. Therefore, we have

$$
|\delta(\omega)| \leq \max \left(\left|\delta_{M C}(\omega)\right|,\left|\delta_{M A}(\omega)\right|\right),
$$

in the transition band of $H_{p}(M \omega)$.

Next, we consider the $\delta(\omega)$ in the frequency range $\left[\frac{2(m+1) \pi-\phi}{M}, \pi\right]$. In this region, all $D(\omega), D_{M A}(\omega)$, and $D_{M C}(\omega)$ are 0 . Substituting these quantities into (6) and ignoring the second order term, we have

$$
\delta(\omega) \simeq \delta_{M C}(\omega) e^{-j \omega M G_{p}}+D_{p}(\omega)\left[\delta_{M A}(\omega)-\delta_{M C}(\omega)\right],
$$

which is exactly the same as (8). Hence the behaviors of the approximation error $\delta(\omega)$ in $\left[\frac{2(m+1) \pi-\phi}{M}, \pi\right]$ in the passband, stopband, and transition band of $H_{p}(M \omega)$ are also described by $(9),(10)$, and (13), respectively.

From the above analysis, we can conclude that $\delta(\omega)$ of $H(\omega)$ in the frequency ranges $\left[0, \frac{2 m \pi-\theta}{M}\right]$ and $\left[\frac{2(m+1) \pi-\phi}{M}, \pi\right]$ is mainly controlled by the approximation error of $F_{M A}^{M}(\omega)$ and $F_{M C}(\omega)$, the complementary property between the $H_{p}\left(z^{M}\right)$ and $H_{c}\left(z^{M}\right)$ makes the approximation error of $H_{p}(\omega)$ has nearly no contribution on $\delta(\omega)$. In other words, for designing $H(\omega)$ to meet the specification in the frequency ranges $\left[0, \frac{2 m \pi-\theta}{M}\right]$ and $\left[\frac{2(m+1) \pi-\phi}{M}, \pi\right]$, all we have to do is properly design the two masking filters $F_{M A}(\omega)$ and $F_{M C}(\omega)$. Consider the problems of designing these two masking filters. Basically, both are design problems in the complex frequency domain. Here, we describe how to use a recently developed WLS algorithm [7] to solve the design problems. In the passband and stopband of these two masking filters, we allow the existence of some "don't care frequency bands" which are weighted less than the other frequency bands [3]. The magnitude of $\delta_{M A}(\omega)$ and $\delta_{M C}(\omega)$ should be $10 \%-15 \%$ smaller then the maximum allow- 
able magnitude of $\delta(\omega)$ in order to neglect the second order error $[3]$.

With the bandedges of $F_{M A}(\omega)$ and $F_{M C}(\omega)$, desired passband group delay $G_{m}$, and suitable minimax weighting function $W_{m x}(\omega)$, the design problem can be formulated as

$$
\text { Minimize }\left\|W_{m x}(\omega)\left(\sum_{n=0}^{N_{m}-1} f_{m}(n) \exp (-j n \omega)-D_{M}(\omega)\right)\right\|
$$

where $\|\cdot\|$ denotes the Chebyshev norm. $D_{M}(\omega)$ is the desired frequency response of the masking filter, in the passhand $D_{M}(\omega)=$ $e^{-j \omega G_{m}}$ and in the stopband $D_{M}(\omega)=0$. Since using the least square criterion will result in a Chebyshev norm minimization if a suitable least square error weighting function can be found, we express the design problem as the minimization of the following weighted frequency respose error

$$
J=\sum_{k} W_{l s}\left(\omega_{k}\right)\left|\sum_{n=0}^{N_{m}-1} f_{m}(n) \exp \left(-j n \omega_{k}\right)-D_{M}\left(\omega_{k}\right)\right|^{2}
$$

where $\omega_{k}$ denotes the $k$ th point on a dense grid of frequency in evaluating the weighted least squares error $J$. Substituting $D_{M}\left(\omega_{k}\right)=P\left(\omega_{k}\right)+j Q\left(\omega_{k}\right)$ into $(16)$, then taking the derivatives of (16) with respect to filter coefficients $f_{m}(n), n=0, \cdots, N_{m}-1$, and setting these derivatives to zero, we obtain the optimal solution which minimizes (16) given by

$$
\begin{aligned}
\mathbf{f}_{\mathbf{m}} & =\left[f_{m}(0), f_{m}(1), \cdots, f_{m}\left(N_{m}-1\right)\right]^{T} \\
& =\mathbf{R}_{\mathbf{0}}^{\prime-1} \mathbf{z},
\end{aligned}
$$

where $\mathbf{R}_{\mathbf{0}}^{\prime}$ is an $N_{m} \times N_{m}$ square matrix with its $(i, j)$ th element given by

$$
\mathbf{R}_{0}^{\prime}(i, j)=\sum_{k} W_{l s}\left(\omega_{k}\right) \cos \left((i-j) \omega_{k}\right),
$$

and $\mathbf{z}^{\prime}$ is an $N_{m} \times 1$ column vector with its element given by

$$
\left.\mathbf{z}^{\prime}(i)=\sum_{k} W_{l_{s}}\left(\omega_{k}\right)\left(P\left(\omega_{k}\right) \cos i \omega_{k}-Q\left(\omega_{k}\right) \sin i \omega_{k}\right)\right),
$$

The structure of $\mathbf{R}_{\mathbf{0}}^{\prime}(i, j)$ given in (18) reveals that $\mathbf{R}_{\mathbf{0}}^{\prime}$ is a Toeplitz and symmetric matrix. Hence, we can solve (17) very efficiently by using a variation of the well-known Levinson algorithm. The suitable least square weighting function $W_{l s}(\omega)$ required for achieving the minimax design can be found by utilizing the weight adjusting algorithm presented in [7].

\section{Design of the equalizer $H_{p}\left(z^{M}\right)$}

When $F_{M A}(\omega)$ and $F_{M C}(\omega)$ have been properly designed, the remaining undetermined regions of $H(\omega)$ are those corresponding to $\left(\frac{2 m \pi-\theta}{M}, \omega_{p}\right]$ and $\left[\omega_{s}, \frac{2(m+1) \pi-\phi}{M}\right)$, which are the regions near the transition band of $H(\omega)$. In these regions, $\delta(\omega)$ is given by (6). $D_{M A}(\omega)+\delta_{M A}(\omega)=F_{M A}(\omega)$ and $D_{M C}(\omega)+\delta_{M C}(\omega)=F_{M C}(\omega)$ are two known functions. Hence, we can use the coefficients of $H_{\mathrm{p}}(z)$ to make $H(\omega)$ also satisfy the desired specifications in these regions. Thus, the design problem is to find the coefficients of $H_{p}(z)$ to minimize the weighted Chebyshev norm of $\delta(\omega)$, i.e.,

$$
\begin{array}{ll}
\text { Minimize } \| \quad & W_{m x}(\omega)\left[H_{p}(M \omega)\left(F_{M A}(\omega)-F_{M C}(\omega)\right)+\right. \\
& \left.F_{M C}(\omega) e^{-j \omega M G_{p}}-D(\omega)\right] \| .
\end{array}
$$

Let $F_{M A}(\omega)-F_{M C}(\omega)$ be denoted as $F_{M}(\omega)$ and $D(\omega)-e^{-j \omega M G_{p}}$ be denoted as $D_{t}(\omega),(20)$ becomes

$$
\text { Minimize }\left\|W_{m x}(\omega)\left[H_{p}(M \omega) F_{M}(\omega)-D_{t}(\omega)\right]\right\| \text {. }
$$

Note that the role of $H_{p}(M \omega)$ is to equalize the frequency response $F_{M}(\omega)$ to make the overall response $H_{p}(M \omega) F_{M}(\omega)$ approximate $D_{t}(\omega)$ in the minimax sense. Based on the use of the WLS algorithm to design the equalizer $H_{p}\left(z^{M}\right)$, the design problem of (21) can be expressed as the minimization of the following weighted frequency response error

$$
J^{\prime}=\sum_{k} W_{l s}\left(\omega_{k}\right)\left|F_{M}\left(\omega_{k}\right) \sum_{n=0}^{N-1} h_{p}(n) \exp \left(-j M \omega_{k} n\right)-D_{t}\left(\omega_{k}\right)\right|^{2}
$$

where $\omega_{k}$ denotes the kth point on a dense grid of frequency in $\left(\frac{2 m \pi-\theta}{M}, \omega_{p}\right]$ and $\left[\omega_{s}, \frac{2(m+1) \pi-\phi}{M}\right)$ for evaluating the weighted least squares error $J^{\prime}$. Substituting $D_{t}\left(\omega_{k}\right)=P\left(\omega_{k}\right)+j Q\left(\omega_{k}\right)$ and $F_{M}\left(\omega_{k}\right)=R\left(\omega_{k}\right)+j I\left(\omega_{k}\right)$ into $(20)$, then taking the derivatives of (22) with respect to filter coefficients $h_{p}(n), n=0, \cdots, N-1$, and setting these derivatives to zero, we obtain the optimal solution which minimizes (22) given by

$$
\begin{aligned}
\mathbf{h}_{\mathbf{p}} & =\left[h_{p}(0), h_{p}(1), \cdots, h_{p}(N-1)\right]^{T} \\
& =\mathbf{R}_{\mathbf{0}}^{-\mathbf{1}} \mathbf{z},
\end{aligned}
$$

where $\mathbf{R}_{0}$ is an $N \times N$ square matrix with its $(i, j)$ th element given by

$$
\mathbf{R}_{\mathbf{0}}(i, j)=\sum_{k} W_{l s}\left(\omega_{k}\right)\left(R^{2}\left(\omega_{k}\right)+I^{2}\left(\omega_{k}\right)\right) \cos \left((i-j) M \omega_{k}\right),
$$

and $\mathbf{z}$ is an $N \times 1$ column vector with its element given by

$$
\begin{aligned}
& \mathbf{z}(i)=\sum_{k} W_{l s}\left(\omega_{k}\right) \\
& \left(P\left(\omega_{k}\right)\left(R\left(\omega_{k}\right) \cos i M \omega_{k}+I\left(\omega_{k}\right) \sin i M \omega_{k}\right)+\right. \\
& \left.Q\left(\omega_{k}\right)\left(I\left(\omega_{k}\right) \cos i M \omega_{k}-R\left(\omega_{k}\right) \sin i M \omega_{k}\right)\right),
\end{aligned}
$$

Again, the structure of $\mathbf{R}_{\mathbf{0}}(i, j)$ given in (24) reveals that $\mathbf{R}_{\mathbf{0}}$ is also a Toeplitz and symmetric matrix. Hence, we can also solve (23) very efficiently by using a variation of the well-known Levinson algorithm. The suitable least square weighting function $W_{l s}(\omega)$ required for achieving the minimax design can also be found by utilizing the weight adjusting algorithm presented in [7].

\section{Computer Simulation Examples}

In this section, we present several design examples to illustrate our design method. All of the designs are performed on a MICRO-VAX MV 3600 minicomputer.

\section{Example 1}

In this example, we design a continuous coefficient lowpass filter with passband edge $\omega_{p}=0.6 \pi$, stopband edge $\omega_{s}=0.61 \pi$, and the Chebyshev approximation error $\|\delta(\omega)\|$ is less than or equal to 0.01 . The desired passband group delay is 120 (if we design a linear phase system to meet such specification, the group delay is about 200). By using the design procedure we state in Section III, Section IV, and Section V, we have design three subfilters $F_{M A}(z)$, $F_{M C}(z)$, and $H_{p}(z)$, and their length are 46,35 , and 51 , respectively. The magnitude response and the unwrapped passband phase response are shown in Fig. 3.

\section{Conclusion}

In this paper, we have considered the design problem for synthesizing a sharp FIR filter with prescribed group delay. Based on a WLS algorithm, we extend the frequency response masking technique to the complex frequency domain to solve the problem. In the proposed method, we first search the suitable interpolating factor to minimize the complexity of the overall system. Then, we determine the design specifications of the required masking filters and design them. The specifications include the desired frequency response, suitable minimax error weighting function, and allowable peak ripple value. After designing the masking filters, we also use the WLS algorithm for designing the shaping filter to equalize the overall response. It has been shown by computer simulation examples that a sharp FIR filter with prescribed group delay can be easily designed by using the proposed method.

\section{References}

[1] Y. Neuvo, C.-Y. Dong, and S. K. Mitra, "Interpolated finite 
impulse response filters," IEEE Trans. Acoust., Speech, Signal Processing, vol. ASSP-32, pp.563-570, June 1984.

[2] T. Saramäki, Y. Neuvo, and S. K. Mitra, "Design of computationally efficient interpolated FIR filters " IEEE Trans. Circuits Syst., vol. CAS-35, pp. 70-87, Jan. 1988.

[3] Y. C. Lim, "Frequency-response masking approach for the synthesis of sharp linear phase digital filters," IEEE Trans. Circuits Syst., vol. CAS-33, pp. 357-364, Apr. 1986.

[4] J. F. Kaiser, and R. W. Hamming, "Sharpening the response of a symmetric nonrecursive filter by multiple use of the same filter," IEEE Trans. Acoust., Speech, Signal Processing, vol. ASSP-25, pp.415-422, Oct. 1977.

[5] T. Saramäki, "Design of FIR filters as a tapped cascaded interconnection of identical subfilters," IEEE Trans. Circuits Syst., vol. CAS-34, pp. 1011-1029, Sep. 1987.

[6] X. Chen and T. W. Parks, "Design of FIR filters in the complex domain," IEEE Trans. Acoust., Speech, Signal Processing., vol. ASSP-35, pp. 144-153, Jun. 1987.

[7] Y. C. Lim, J. H. Lee, C. K. Chen and R. H. Yang, "A weighted east squares algorithm for quasi-equiripple FIR and IIR digital filter design," IEEE Trans. Signal Processing., vol. ASSP-40, pp. 551-558, Mar. 1992.

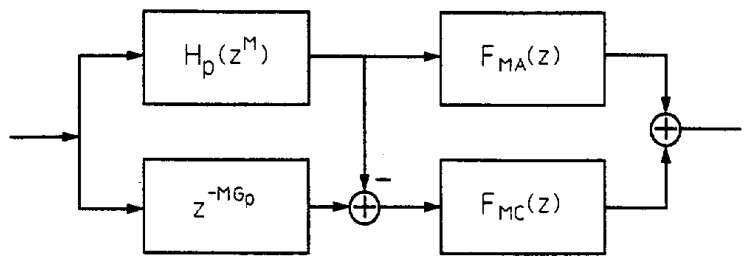

Fig. 1
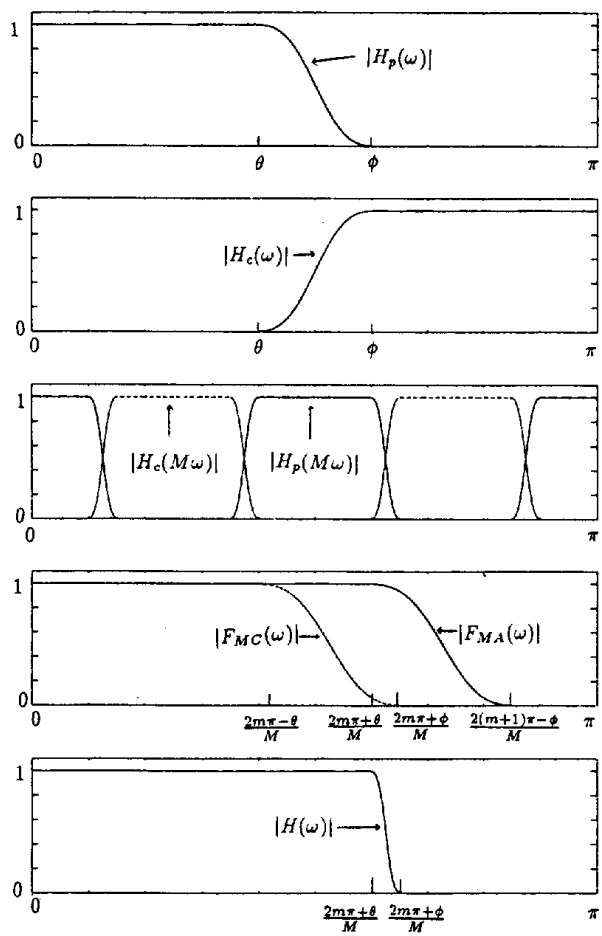

Fig. 2
TABLE I Bandedges of subfilters for Case I and Case II

\begin{tabular}{||c||c|c|}
\hline \hline & Case I & Case II \\
\hline \hline$m$ & {$\left[\omega_{p} M / 2 \pi\right]$} & {$\left[\omega_{s} M / 2 \pi\right]$} \\
\hline$\theta$ & $\omega_{p} M-2 m \pi$ & $2 m \pi-\omega_{s} M$ \\
\hline$\phi$ & $\omega_{s} M-2 m \pi$ & $2 m \pi-\omega_{p} M$ \\
\hline$\omega_{A p}$ & $\frac{2 m \pi+\theta}{M}$ & $\frac{2(m-1) \pi+\phi}{M}$ \\
\hline$\omega_{A s}$ & $\frac{2(m+1) \pi-\phi}{M}$ & $\frac{2 m \pi-\theta}{M}$ \\
\hline$\omega_{C p}$ & $\frac{2 m \pi-\theta}{M}$ & $\frac{2 m \pi-\phi}{M}$ \\
\hline$\omega_{C s}$ & $\frac{2 m \pi+\phi}{M}$ & $\frac{2 m \pi+\theta}{M}$ \\
\hline
\end{tabular}

$\lfloor x\rfloor$ stands for the largest integer less than $x$.

$\lceil x\rceil$ stands for the smallest integer larger than $x$.
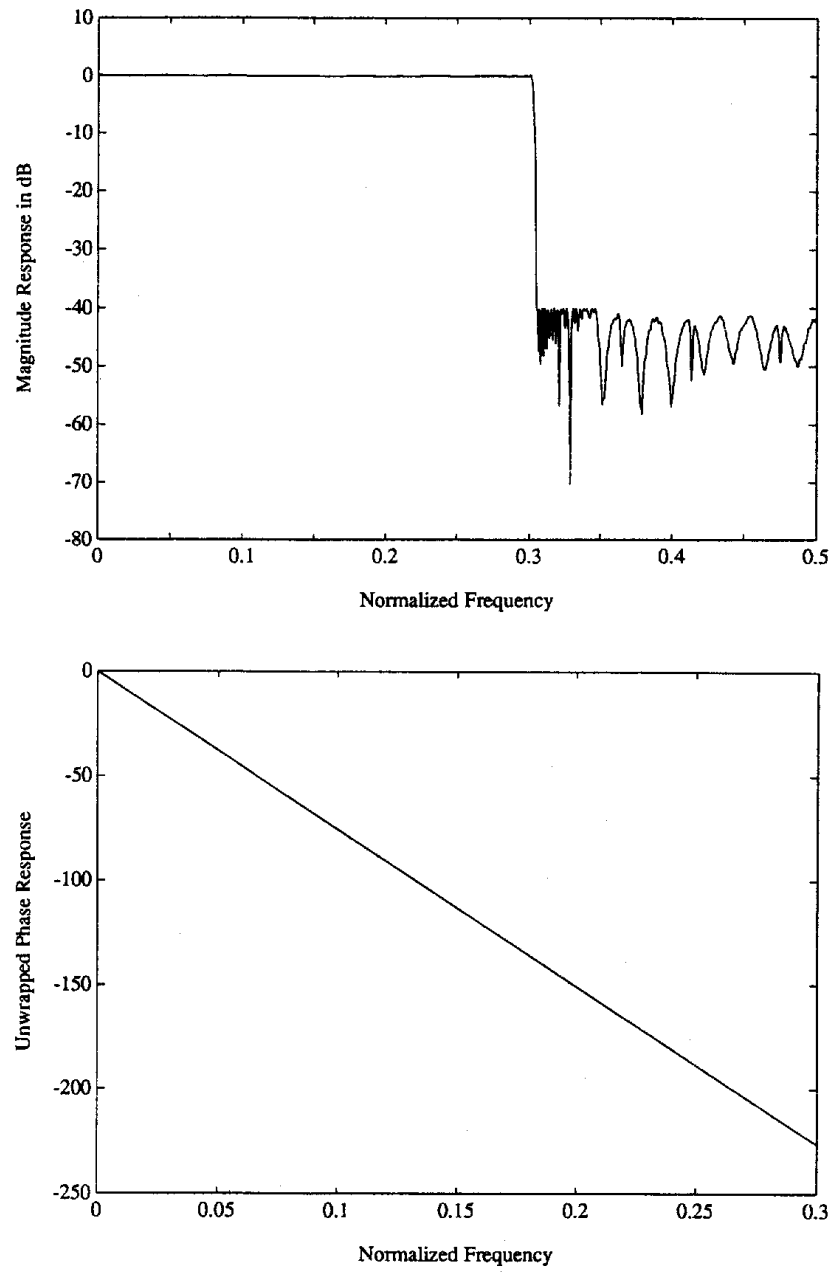

Fig. 3 